\title{
Laparoscopic Pelvic Autonomic Nerve-Preserving Surgery for Sigmoid Colon Cancer
}

\author{
Jin-Tung Liang, MD, PhD, Hong-Shiee Lai, MD, PhD, Po-Huang Lee, MD, PhD, \\ and King-Jen Chang, MD, PhD
}

Division of Colorectal Surgery, Department of Surgery, National Taiwan University Hospital and College of Medicine, No. 7, Chung-Shan South Road, Taipei, Taiwan

\begin{abstract}
Background: To test the feasibility of laparoscopic approach in performing the simultaneous pelvic autonomic nerve preservation during standard anterior resection of sigmoid colon cancer.

Methods: Patients meeting appropriate eligibility criteria were recruited for the present study. The surgical procedures are shown in the video. The genitourinary function was evaluated on the basis of validated questionnaires including International Prostate Symptom Score (IPSS), International Index of Erectile Function (IIEF), and Female Sexual Function Index (FSFI).

Results: A total of 112 patients (tumor, node, metastasis system stage $\mathrm{I}, \mathrm{n}=8$; stage II, $\mathrm{n}=54$; stage III, $\mathrm{n}=50$; male, $\mathrm{n}=58$; female, $\mathrm{n}=54$; age [mean \pm standard deviation], $55.8 \pm 6.4$ years) with good baseline genitourinary function were operated on with the intent of total preservation of pelvic autonomic nerves and curative resection of sigmoid colon cancer. The patients were prospectively followed (median time of follow-up, 18 months; range, 6-30 months). In patients with a successful nerve-preserving surgery $(96.4 \%, \mathrm{n}=108), 104$ patients completed the evaluation of urinary function. The median duration for indwelling urine Foley catheter was 3.0 days (range, 1.0-7.0 days). The voiding function after removal of the urine Foley catheter was good (IPSS, 0-7) in 98 $(94.2 \%)$ patients, fair (IPSS, 8-14) in $5(4.8 \%)$, and poor (IPSS, 15-35) in $1(1.0 \%)$. Before and after nerve-preserving surgery, there were no significant changes of IPSS scores $(3.20 \pm 1.72$ vs. $3.68 \pm 2.82, P=.075$, paired $t$-test) in the present patient series. Fortyfour male patients completed the postoperative evaluation of sexual function, and ejaculation was ranked as good in $40(90.9 \%)$, fair (decrease in ejaculatory amounts) in 3 $(6.8 \%)$, and poor (retrograde ejaculation, failure of ejaculation) in $1(2.3 \%)$, whereas the potency was good (IIEF, 60-75) in $41(93.2 \%)$, fair (IIEF, 44-59) in $2(4.5 \%)$, and poor (IIEF, 5-43) in $1(2.3 \%)$. Moreover, before and after a successful nerve-preserving operation, there were no significant changes of IIEF scores $(72.4 \pm 4.6$ vs. $70.3 \pm 8.4$, $P=.082$, paired $t$-test $)$. For female patients $(\mathrm{n}=42)$, the postoperative sexual function was ranked as good (FSFI score, 76-95) in $36(85.7 \%)$, fair (FSFI, 58-75) in $4(9.5 \%)$, and poor (FSFI, 4-57) in $2(4.8 \%)$. Furthermore, there were no significant changes of FSFI scores $(89.0 \pm 9.2$ vs. $85.4 \pm 16.4, P=.122$, paired $t$-test) before and after successful nerve-preserving surgery.
\end{abstract}

Published online March 26, 2008.

Electronic supplementary material: The online version of this article (doi:10.1245/s10434-008-9861-x) contains supplementary material, which is available to authorized users.

Address correspondence and reprint requests to: Jin-Tung Liang, MD, PhD; E-mail: jintung@ntu.edu.tw

Published by Springer Science+Business Media, LLC @ 2008 The Society of Surgical Oncology, Inc. 
Conclusions: Under laparoscopy, we can clearly identify and preserve the pelvic autonomic nerves to retain genitourinary function in most patients undergoing oncologic resection of sigmoid colon cancer.

Key Words: Laparoscopic surgery—Sigmoid colon cancer-Pelvic autonomic nerve.

Preservation of pelvic autonomic nerves to retain genitourinary function without compromise of oncologic clearance of tumor has been extensively studied in rectal cancer surgery. ${ }^{1-12}$ However, there is still not much information on sexual and bladder function after the curative resection of sigmoid colon cancer. Theoretically, the pelvic autonomic nervepreserving surgery is technically much easier during a sigmoid colon resection, as apposed to rectal surgery, because the pelvic deep-seated neural structures, such as pelvic plexus (inferior hypogastric plexus), lateral ligament, neurovascular bundles of Walsh, and periprostate plexus, were not within the boundary of dissection. ${ }^{13-17}$ It is therefore our opinion that during the anterior resection of sigmoid colon cancer, preservation of autonomic nerves is a priority - that is, autonomic nerve preservation is appropriate unless there are oncologic reasons for the resection of nerves, e.g., the nerves were directly involved by cancer, in terms of better postoperative genitourinary function for the patients. Recently, more and more sigmoid colon cancers were resected laparoscopically. ${ }^{18-20}$ Therefore, we conducted the present study to test the feasibility of laparoscopic approach in performing the total pelvic autonomic nerve preservation during the standard oncologic resection of sigmoid colon cancer. We hypothesized that given a well-illuminated magnified view by laparoscopy, the autonomic nerves can be well protected from inadvertent surgical damage.

\section{PATIENTS AND METHODS}

Patients meeting the following eligibility criteria were recruited for the present study. The inclusion criteria were: (1) curative and elective surgery; (2) sigmoid adenocarcinoma with tumor location generally 15 to $40 \mathrm{~cm}$ above anal verge; (3) American Society of Anesthesiology function class I to class III patients; (4) age between 18 to 65 years. The exclusion criteria were: (1) emergency or urgent surgery; (2) evidence of tumor invasion to adjacent organs or distant metastasis; (3) previous major abdominal or pelvic surgery; (4) abnormal preoperative baseline genitourinary function on the basis of validated questionnaire-based interview. ${ }^{10,21,22}$ Before entering the present study, all patients were well explained regarding the surgical procedures, and informed consents were obtained. The present study was approved by the Institutional Review board of National Taiwan University Hospital and was designated as NTUH 93S-024.

\section{Surgical Strategy}

The related neuroanatomy for the nerve-preserving surgery of sigmoid colon cancer is shown in Fig. 1A. The preservation of autonomic nerves was focused on the following three anatomic sites: (1) inferior mesenteric plexus (Fig. 1B); (2) superior hypogastric plexus (Fig. 1C); and (3) the paired hypogastric nerves (Fig. 1D). The details of surgical procedures were well recorded and are shown in the associated video.

To ensure the visualization and preservation of the nerves at all three critical points, we used a specific point-by-point checklist during the operations. The dissection commences at the medial side of sigmoid mesentery at the level of pelvic promontory. By gentle tenting of the sigmoid mesocolon ventrally, the superior hypogastric plexus was identified and preserved at the interiliac trigone just below the peritoneum. Subsequently, the dissection was continued upward along the ventral side of abdominal aorta. The preaortic plexus were then preserved by sparing the preaortic connective tissue. Thereafter, the medial-to-lateral laparoscopic dissection along the areolar tissue between sigmoid mesocolon and Gerota fascia was performed. The medial approach is well adapted for laparoscopy because it provides the working space and demands the least handling of sigmoid colon. ${ }^{23}$

During the mobilization of sigmoid mesocolon, we did not identify the ureter because the loose connective tissue between Gerota fascia and sigmoid mesocolon is a natural dissection plane. Normally, the ureter and gonadal vessels are covered by the Gerota fascia, and therefore, if the ureter is clearly or intentionally identified, it means that the Gerota fascia is opened and the dissection plane may be imprecise. By simultaneous upward and medial-to-lateral dissec- 

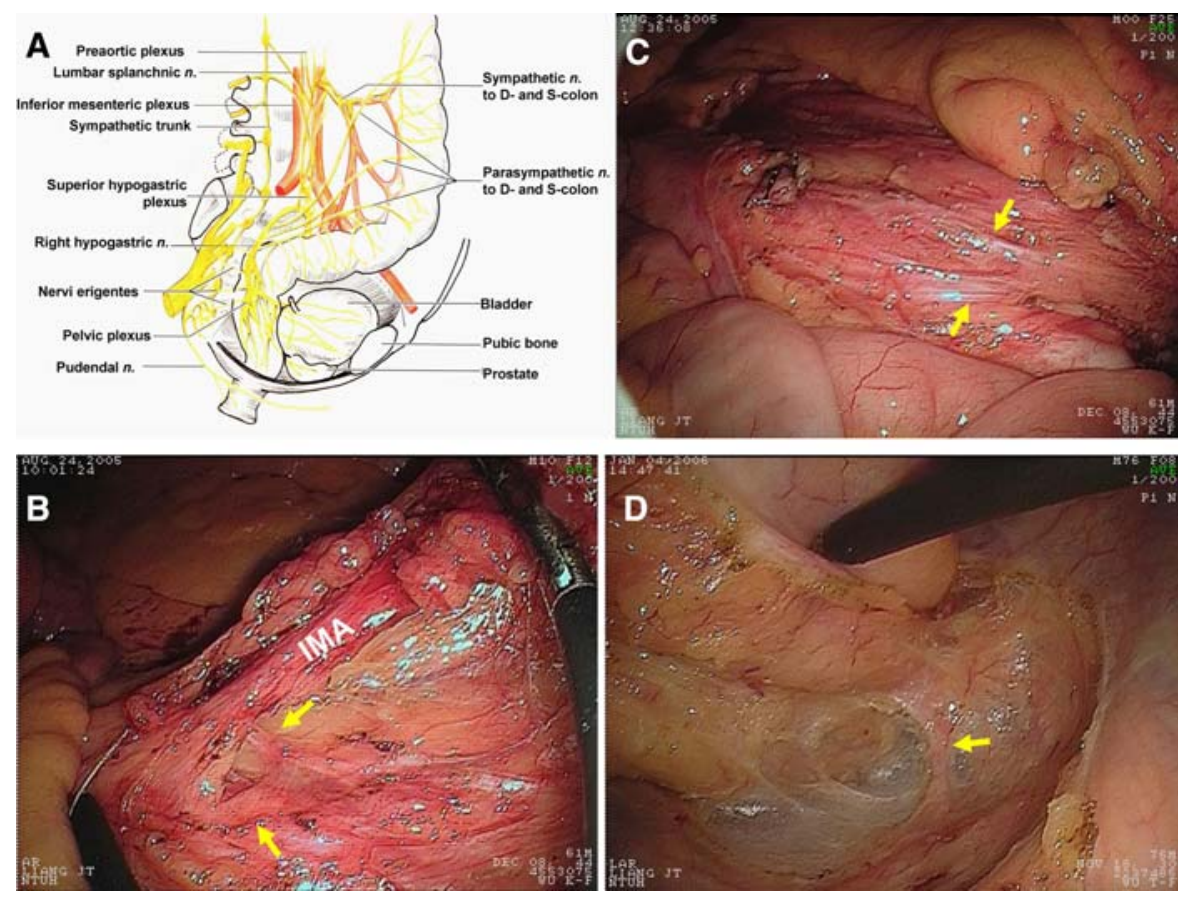

FIG. 1. (A) Surgical anatomy of pelvic anatomic nerves related to the nerve-preserving surgery of sigmoid colon cancer. (B) The inferior mesenteric plexus (yellow arrow) encircles the root of inferior mesenteric artery (IMA). (C) The superior hypogastric plexus overlying the interiliac trigone area. (D) The right hypogastric nerve travels across the presacral areolar tissue and then adheres to the proper rectal fascia.

tion, we subsequently reach the junction of inferior mesenteric artery (IMA) and abdominal aorta. The IMA was divided 1 to $2 \mathrm{~cm}$ distal to its origin, or just distal to the left colic branch. This is because the sympathetic fibers encircle the IMA at the level of IMA's origin from abdominal aorta. If the nerves are not adequately cleared from "behind" the IMA, they can be easily damaged at this point (Fig. 1B). Subsequently, the "holy plane" at the transition of the mesosigmoid to the mesorectum was meticulously dissected to progressively displace the hypogastric nerves dorsally and laterally (Fig. 1D), and thus preserve them.

\section{Assessment of Urinary Function}

Urinary function was evaluated on the basis of questionnaire-based interview of patients preoperatively and 3 months after surgery. In patients with major surgical complications, for example, anastomotic leakage, the urinary function was evaluated 3 months after recovery from surgical morbidity, i.e., 3 months after the closure of defunctioning ileostoma. The preoperative functional questionnaire was based on the recollection of function before the development of symptoms from sigmoid colon cancer. Routinely, the urine Foley catheter was inserted for the patients immediately before the operation. In the postoperative period, the date for urine removal of the Foley catheter and the nature of any voiding problems, such as those requiring recatheterization, were recorded. The duration of indwelling urine Foley catheter was counted from the time of insertion to the time of its removal. The questionnaire used was based on International Prostate Symptom Score (IPSS), the parameters of which included incomplete bladder emptying, frequency, intermittency, urgency, weak stream, straining, and nocturia. Further subdivisions were added to the seven items. ${ }^{10}$ The scoring system for voiding function described above was based on a 0 to 5 scale, as follows: 0 , not at all; 1 , less than one time in five; 2, less than half the time; 3, approximately half the time; 4 , more than half the time; 5, almost always. IPSS was calculated by adding the item scores. We ranked the urinary function as good (IPSS, 0-7); fair (IPSS, 8-14), and poor (IPSS, 15-35). Any voiding problems recovered within 3 months after surgery was considered to be transient bladder voiding dysfunction; otherwise, the voiding problems were deemed persistent.

\section{Assessment of Sexual Function}

Sexual function was evaluated preoperatively and 6 months after operation or recovery from surgical complications, if present.

In male patients, the sexual function was evaluated on the basis of potency and ejaculation. The ejaculatory function was graded as good, fair (decrease in ejaculatory amounts up to $50 \%$ ), and poor 
(retrograde ejaculation, failure to ejaculate). The grade of potency disorder was measured on the basis of the International Index of Erectile Function (IIEF), which contains five index domains (erectile function, orgasm function, sexual desire, intercourse satisfaction, and overall satisfaction) and 15 items. $^{21}$ Each item of the IIEF is scored on a 5- or 6-point Likert score. Score for each domain are variable, and the total score range is 5 to 75 . We classified the male potency function as good (IIEF, 60-75), fair (IIEF, 44-59), and poor (IIEF, 5-43).

In female patients, sexual function was evaluated on the basis of the Female Sexual Function Index (FSFI), which is a 19-item validated questionnaire that was designed to evaluate female sexual dysfunction, especially female sexual arousal disorder. ${ }^{22}$ The scale is divided into six domains: desire, arousal, lubrication, orgasm, satisfaction, and pain. Each item is scored with a 5- or 6- point Likert scale. The scales for each domain are variable, and the total score range is 4 to 95 . For the present study, we classified the female sexual function as good (FSFI, 76-95), fair (FSFI, 58-75), and poor (FSFI, 4-57). Furthermore, the specific sexual problems in women such as libido, arousal, lubrication, orgasm, and dyspareunia were investigated.

\section{Data Management}

Data on patients undergoing laparoscopic autonomic nerve-preserving surgery for sigmoid colon cancer between January 2004 and December 2006 were entered into a prospective database of the present study. Until June 2007, the mean time of follow-up for the patients was 18 months, ranging from 6 to 36 months. The genitourinary function of the patients was analyzed by the intention-to-treat principle. Patients were first contacted by telephone by a research assistant who described the nature of the project. Those who consented to the present study were given a questionnaire-based interview by the research assistant. The data were managed by a statistician. To prevent bias of evaluation, the research assistant was not told which surgical procedure was performed for each individual patient.

Because genitourinary function is influenced by preoperative function and numerous other variables, including the emotional and physiological status of the patients, as well as other influences such as chemotherapy, each patient was used as his or her own control in evaluating the change of function before and after surgery. The two sets of measurements on the same patient were compared, and the $P$ value was
TABLE 1. Demographics, clinicopathologic data, and surgical outcomes of patients undergoing laparoscopic autonomic nerve-preserving surgery for sigmoid colon cancer

$$
(n=112)
$$

\begin{tabular}{ll}
\hline Parameter & \multicolumn{1}{c}{ Value $^{a}$} \\
\hline Age (years) & $55.8 \pm 6.4$ \\
Sex (M/F) & $58 / 54$ \\
Body mass index (weight/height ${ }^{2}$, & \\
$\left.\quad \mathrm{kg} / \mathrm{m}^{2}\right)$, mean (range) & \\
Male & $23.4(20.3 \sim 31.6)$ \\
Female & $24.8(22.0 \sim 32.5)$ \\
ASA class & 62 \\
I & 46 \\
II & 4 \\
III & \\
TME stage (pathologic) & 8 \\
I & 54 \\
II & 50 \\
III & $184.4 \pm 44.8$ \\
Operative time (minutes) & $.9 \%(\mathrm{n}=1)$ \\
Conversion rate & $84.5 \pm 24.0$ \\
Blood loss (mL) & $48.0 \pm 8.4$ \\
Postoperative ileus (hours) & $8.0 \pm 2.0$ \\
Hospitalization (days) & $3.5 \pm .4$ \\
Postoperative pain (visual analog scale) & \\
Postoperative complication & $7.1 \%(\mathrm{n}=8)$ \\
$\quad$ Total & 4 \\
Wound infection & 1 \\
Deep vein thrombosis & 1 \\
Pneumonia & 2 \\
Anastomotic leakage & $14.4 \pm 4.0$ \\
No. of lymph nodes collected & $8.0 \%(\mathrm{n}=9)$ \\
Tumor recurrence rate & \\
\hline
\end{tabular}

ASA, American Society of Anesthesiology; TNM, tumor, node, metastasis system.

${ }^{a}$ Continuous data are presented as mean $\pm \mathrm{SD}$.

calculated from paired $t$-tests. A statistically significant level of difference was set at a $P$ value of $\leq .05$.

\section{RESULTS}

A total of 112 patients were operated on with the intent of total preservation of pelvic autonomic nerves and curative resection of sigmoid colon cancer. The demographics and clinicopathologic data are shown in Table 1 . The nerve-preserving surgery was considered as failed in four patients when the videos of the surgical procedures were rescrutinized by coauthor colorectal surgeons and one general surgeon. The nerve-preserving anterior resection of sigmoid colon was performed with acceptable operation time, little blood loss, and low morbidity. The number of dissected lymph node was adequate, and the short-term oncologic result was good.

In patients with successful nerve-preserving surgery $(96.4 \%, \mathrm{n}=108), 104$ patients completed the evaluation of urinary function (Table 2). The median 
TABLE 2. Changes in urinary function before and after surgery for patients with successful nerve-preserving surgery $(n=104)$

\begin{tabular}{llll}
\hline $\begin{array}{l}\text { Urinary function } \\
\text { (range of IPSS) }\end{array}$ & \multicolumn{1}{c}{$\begin{array}{c}\text { Before } \\
\text { operation }\end{array}$} & $\begin{array}{c}\text { After } \\
\text { operation }\end{array}$ & $P$ value $^{a}$ \\
\hline Good (0-7) & 104 & 98 & \\
Fair (8-14) & 0 & 5 & \\
Poor (15-35) & 0 & 1 & \\
IPSS score (mean \pm SD) & $3.20 \pm 1.72$ & $3.68 \pm 2.82$ & .075 \\
\hline \multicolumn{4}{l}{ IPSS, International Prostate Symptom Score. } \\
${ }^{a} P$ value was calculated on the basis of statistics of paired $t$-test.
\end{tabular}

duration of the indwelling urine Foley catheter was 3.0 days, ranging from 1.0 to 7.0 days. Preoperatively, the baseline urinary function of all patients was ranked as good (IPSS, 0-7). However, after postoperative removal of the urine Foley catheter, the ranks of IPSS were changed to good in 98 patients $(94.2 \%)$, fair (IPSS, 8-14) in $5(4.8 \%$ ), and poor (IPSS, 15-35) in $1(1.0 \%)$. There was no significant difference of preoperative and postoperative IPSS score $(P=.075$, paired $t$-test) in patients with successful nerve-preserving surgery. The six patients with fair or poor bladder function experienced only transient dysfunction and recovered thereafter. Forty-four male and 42 female patients who were sexually active before the operation, i.e., whose ejaculatory function, IIEF, and FSFI score were all ranked as good, responded to the assessment of sexual function. Postoperatively, in male patients (Table 3), the ranks of ejaculatory function were changed to good in $40(90.9 \%)$, fair (decrease in ejaculatory amounts) in $3(6.8 \%)$, and poor (retrograde ejaculation, failure of ejaculation) in $1(2.3 \%)$. The grades of the potency were changed to good (IIEF, 60-75) in 41 (93.2\%), fair (IIEF, 44-59) in $2(4.5 \%)$, and poor (IIEF, $5-43)$ in $1(2.3 \%)$. There was no significant difference of preoperative and postoperative IIEF score $(P=.082$, paired $t$-test $)$ in male patients whose pelvic autonomic nerves were successfully preserved during surgery. In female patients (Table 3), the ranks of sexual function were changed to good (FSFI score, 76-95) in $36(85.7 \%)$, fair (FSFI score, $58-75)$ in $4(9.5 \%)$, and poor (FSFI score, $4-57)$ in $2(4.8 \%)$. However, there was no significant change of FSFI score $(P=.122$, paired $t$ test) for female patients before and after successful nerve-preserving surgery. Remarkably, specific sexual problems in women with autonomic nerve preservation included vaginal lubrication in $14.3 \%(\mathrm{n}=6)$, dyspareunia in $9.5 \%(\mathrm{n}=4)$, sexual arousal in $4.7 \%$ $(\mathrm{n}=2)$, and orgasm in $4.7 \%(\mathrm{n}=2)$.

Of the four patients $(3.6 \%)$ who failed the procedures of the nerve-preserving surgery, three were
TABLE 3. Changes in sexual function for male and female patients with successful nerve-preserving surgery

\begin{tabular}{llll}
\hline Function & $\begin{array}{c}\text { Before } \\
\text { Operation }\end{array}$ & $\begin{array}{c}\text { After } \\
\text { Operation }\end{array}$ & $P$ value $^{a}$ \\
\hline Male patients $(\mathrm{n}=44)$ & & & \\
Ejaculatory function & & & \\
$\quad$ Good & 44 & 40 & \\
$\quad$ Fair & 0 & 3 & \\
$\quad$ Poor & 0 & 1 & \\
Erectile function (IIEF Score) & & \\
$\quad$ Good (60-75) & 44 & 41 & \\
$\quad$ Fair (44-59) & 0 & 2 & \\
$\quad$ Poor (5-43) & 0 & 1 & \\
IIEF score (mean \pm SD) & $72.4 \pm 4.6$ & $70.3 \pm 8.4$ & \\
Female patients (n $=42)$ & & & \\
Sexual function (FSFI Score) & 42 & 36 & \\
$\quad$ Good (76-95) & 0 & 4 & \\
$\quad$ Fair (58-75) & 0 & 2 & \\
$\quad$ Poor (4-57) & $89.0 \pm 9.2$ & $85.4 \pm 16.4$ & .122 \\
FSFI score (mean \pm SD) & 89.4
\end{tabular}

IIEF, International Index of Erectile Function; FSFI, Female Sexual Function Index.

${ }^{a} P$ value was calculated on the basis of statistics of paired $t$-test.

female and one was male (Table 4). In them, the mean duration of indwelling urine Foley catheter was 6 days (range, $4-8$ days). Although the genitourinary function for the four patients was normal before surgery, the single male patient's IPSS and IIEF rank declined to the level of "fair" and he experienced retrograde ejaculation after the operation. The IPSS rank of the three female patients changed to the "fair" level in two, but the other patient remained at a "good" level after operation. The sexual function was adversely affected by the failure of nerve-preserving surgery, with a "fair" rank of FSFI in one patient and a "poor" rank in the remaining two patients. All three female patients experienced specific sexual problems, including vaginal lubrication, dyspareunia, sexual arousal, and orgasm.

\section{DISCUSSION}

The present study confirmed our hypothesis that by taking advantage of the optic properties of laparoscopy, we can precisely preserve the pelvic autonomic nerve at the following three anatomic sites: inferior mesenteric plexus, superior hypogastric plexus, and paired hypogastric nerves. However, we failed to preserve the autonomic nerve in four (one male and three female) patients of this case series - a finding that needs to be addressed. After the completion of nerve-preserving surgery, we routinely tented the superior hypogastric plexus to check the total preservation of associated nerves. In our 
TABLE 4. Changes of values in specific domains of the scoring system for evaluation of genitourinary function in patients who failed the procedures of nerve-preserving surgery $(n=4)$

\begin{tabular}{|c|c|c|c|c|c|c|c|c|}
\hline \multirow[b]{2}{*}{ Domain } & \multicolumn{2}{|c|}{ Patient 1 (male) } & \multicolumn{2}{|c|}{ Patient 2 (female) } & \multicolumn{2}{|c|}{ Patient 3 (female) } & \multicolumn{2}{|c|}{ Patient 4 (female) } \\
\hline & Before OP & After OP & Before OP & After OP & Before OP & After OP & Before OP & After OP \\
\hline \multicolumn{9}{|l|}{ IPSS (score range) } \\
\hline Total & 0 & 10 & 0 & 2 & 0 & 9 & 0 & 12 \\
\hline 1. Incomplete emptying $(0-5)$ & 0 & 2 & 0 & 1 & 0 & 4 & 0 & 4 \\
\hline 2. Frequency $(0-5)$ & 0 & 2 & 0 & 1 & 0 & 1 & 0 & 2 \\
\hline 3. Intermittency $(0-5)$ & 0 & 1 & 0 & 0 & 0 & 2 & 0 & 2 \\
\hline 4. Urgency $(0-5)$ & 0 & 1 & 0 & 0 & 0 & 0 & 0 & 1 \\
\hline 5. Week stream $(0-5)$ & 0 & 1 & 0 & 0 & 0 & 0 & 0 & 0 \\
\hline 6. Straining $(0-5)$ & 0 & 2 & 0 & 0 & 0 & 2 & 0 & 3 \\
\hline 7. Nocturia $(0-5)$ & 0 & 1 & 0 & 0 & 0 & 0 & 0 & 0 \\
\hline \multicolumn{9}{|l|}{ IIEF (score range) } \\
\hline Total & 75 & 44 & - & - & - & - & - & - \\
\hline 1. Erection function $(1-30)$ & 30 & 18 & - & - & - & - & - & - \\
\hline 2. Orgasmic function $(0-10)$ & 10 & 6 & - & - & - & - & - & - \\
\hline 3. Sexual desire $(2-10)$ & 10 & 6 & - & - & - & - & - & - \\
\hline 4. Intercourse satisfaction $(0-15)$ & 15 & 7 & - & - & - & - & - & - \\
\hline 5. Overall satisfaction $(2-10)$ & 10 & 7 & - & - & - & - & - & - \\
\hline \multicolumn{9}{|l|}{ FSFI } \\
\hline Total & - & - & 95 & 58 & 95 & 27 & 95 & 32 \\
\hline 1. Desire $(2-10)$ & - & - & 10 & 8 & 10 & 4 & 10 & 5 \\
\hline 2. Arousal (0-20) & - & - & 20 & 12 & 20 & 5 & 20 & 6 \\
\hline 3. Lubrication $(0-20)$ & - & - & 20 & 10 & 20 & 6 & 20 & 6 \\
\hline 4. Orgasm $(0-15)$ & - & - & 15 & 9 & 15 & 3 & 15 & 4 \\
\hline 5. Satisfaction $(2-15)$ & - & - & 15 & 9 & 15 & 3 & 15 & 5 \\
\hline 6. Pain $(0-15)$ & - & - & 15 & 10 & 15 & 6 & 15 & 6 \\
\hline
\end{tabular}

IIEF, International Index of Erectile Function; FSFI, Female Sexual Function Index.

scrutiny of the surgical videos, we found that the mechanisms of nerve injury for the three female patients were the same: the nerve injuries were all located at the level of the hypogastric nerves and were bilateral. In our experience, the fibers of pelvic autonomic nerve were generally thinner in female that than in male patients. Furthermore, anatomically, the paired hypogastric nerves run downward, close to or tethering the proper rectal fascia along the pelvic wall (Fig. 1D). At this point, the right-sided hypogastric nerves of the three female patients were subjected to avulsion injury. On the other hand, the left-side hypogastric nerves of these three female patients were mistaken as pelvic fibrotic bands and were cauterized. We thought that some female patients might be predisposed to chronic pelvic inflammation, thus blurring the pelvic dissection plane, and therefore the hypogastric nerves and retroperitoneal fibrotic bands could not be clearly differentiated, even under the well-illuminated and magnified view of laparoscopy. In the only male case of autonomic nerve injury, we found that the patient's body mass index (BMI) was $32.0 \mathrm{~kg} / \mathrm{m}^{2}$. During the surgery, the hypogastric nerve was coated by fatty tissues and was inadvertently severed bilaterally. Because obese patients (BMI $\left.>30 \mathrm{~kg} / \mathrm{m}^{2}\right)$ represented only $6(10.3 \%)$ of the 58 male and $4(7.4 \%)$ of 54 female patients in this case series and nerve injury related to obesity occurred in only 1 male patient, we therefore were not sure whether high BMI was a risk factor for nerve injury. Further recruitment of more obese patients for study will be necessary to solve this interesting question.

Remarkably, CaverMap Surgical Aid (CM; Blue Torch Medical Technologies, Inc., Ashland, MA) has been recommended to assist surgeons in intraoperatively localizing the autonomic nerves, ultimately leading to improved preservation of autonomic nerves and sexual function. ${ }^{24} \mathrm{We}$ thought that CaveMap might be useful in case the nerve-preserving procedures were for the treatment of rectal cancer, in which case the neural structures to be preserved were deep-seated in the pelvis. However, for the oncologic resection of sigmoid colon cancer, the autonomic nerves at the above-mentioned three critical anatomic points were usually be clearly identified. In the rare cases when exact identification of the autonomic nerves was difficult, we recommended that the surgeons find the precise dissection plane between sigmoid mesentery and retroperitoneal tissue, and thus a plate of nerve-containing tissue overlying the abdominal aorta and interiliac trigone 
area can be left intact. On the other hand, the present study showed that laparoscopic nerve-preserving surgery for sigmoid colon cancer was technically feasible and could achieve function-preserving outcomes as good as those of traditional open surgery. ${ }^{10,11}$ However, the functional results of most reported series to date were based on the patients with rectal cancer undergoing open laparotomy. Therefore, further randomized studies in this respect that focus on sigmoid colon cancer are necessary.

Neurophysiologically, the internal urethral orifice is innervated by sympathetic signals and has dual functions: one to ensure urinary continence, and the other to prevent backflow of semen during ejaculation. The present study provided the insights into the functional significance of the internal urethral orifice by the clinical observation that the female patients manifested some degree of urinary incontinence and the male patients experienced retrograde ejaculation as a result of the failed preservation of bilateral hypogastric nerves. However, the influence of damage of pelvic sympathetic nerves on female sexual function has been ambiguous but generally considered because it would most likely result in impairment of vaginal lubrication. The manifestation of specific sexual problems for the three female patients with inadvertent sympathectomy supported this hypothesis. Remarkably, our previous study on the canine modal showed that when one side of hypogastric nerve was severed, the other side of hypogastric nerve could compensate for the genitourinary functional loss to a normal level. ${ }^{15}$ To clarify the role of pelvic sympathetics in the human genitourinary function, further recruitment of more patients with iatrogenic sympathetic nerve injury for study is required. Because the intentional severance of sympathetic nerve during the surgical practice is not ethical, we think that further study subjects will be focused on patients with an advanced rectosigmoid cancer in which unilateral or bilateral sympathectomy was a necessity for the radical resection of cancer.

In the present study, 6 patients $(5.8 \%)$ had transient voiding disorder, $4(9.1 \%)$ of 44 male patients had ejaculatory disorder, and up to $14.3 \%$ (6 of 42 ) female patients had sexual problems after the operation, even though their sympathetic nerves were successfully preserved during surgery. Our explanations for this phenomenon were as follows: first, anatomically, the inferior mesenteric plexus, thoracolumbar sympathetic splanchnic nerves, and superior hypogastric plexus were grossly manifested as a continuous plate of weblike connective tissues underlying the root of IMA and overlying the lower abdominal aortic and the interiliac trigone area. Therefore, some minor partial injury of nerve fibers may not be detected during surgery. Second, the retroperitoneal dissection by electrocauterization sometimes causes side injury and/or ischemia change of nerve fibers and thus compromised the neurophysiologic function. Third, the sexual function is influenced by various psychosocial factors. For example, the alteration of body image and/or bowel function after the operation may also have negative effect on sexual function.

Genitourinary functional disorders are generally subjective symptoms, and their measurement, either by questionnaire-based interviews or laboratory manometric and volumetric studies, are vulnerable to evaluation bias. Therefore, before the translation of technical feasibility of nerve-preserving surgery into the retaining of normal genitourinary function, the methodology used for the evaluation of functional outcomes in the present study needs to be further addressed. First, we used IPSS, IIEF, and FSFI to measure the genitourinary function of patients. These three kinds of validated questionnaires have been generally considered to be highly sensitive, and they have been widely adopted internationally for the study of genitourinary function. ${ }^{21,22}$ Second, in this study, both the patients and the assessors were informed of the intent to preserve the sympathetic nerves during sigmoidectomy but actually had no idea whether the nerve-preserving procedure was successful. The blinding of both assessors and patients to the surgical procedure can lessen the placebo effect. Third, the genitourinary function varies at different time points of evaluation. In this study, the evaluation of postoperative genitourinary function was conducted at the standardized time points before and after the operation. However, the preoperative baseline data was based on patient recall of genitourinary function before the appearance of bowel symptoms. The evaluation of baseline genitourinary function was therefore inevitably subject to methodology limitations, i.e., the potential recall bias. However, to validate the preoperative baseline data of patients, the spouses or sexual partners of some selected patients were also interviewed. We emphasize that patients were included in this study only when their normal baseline genitourinary function was ensured.

In conclusion, the genitourinary function of patients can be successfully preserved with good technical efficiency during laparoscopic sigmoidectomy. The present study should facilitate further study regarding the impact of preservation of genitourinary function on 
the postoperative quality of life for patients with sigmoid colon cancer.

\section{REFERENCES}

1. Moriya Y. Pelvic node dissection with autonomic nerve sparing for invasive lower rectal cancer: Japanese experience. In: Wanebo HJ, editor. Colorectal cancer. St Louis, MO: MosbyYear Book, 1993; p. 274-89.

2. Havenga K, Maas CP, DeRuiter MC, et al. Avoiding longterm disturbance to bladder and sexual function in pelvic surgery, particularly with rectal cancer. Semin Surg Oncol 2000; 18:235-43.

3. Hendren SK, O'Connor BI, Liu M, et al. Prevalence of male and female sexual dysfunction is high following surgery for rectal cancer. Ann Surg 2005; 242:212-23.

4. Maeda K, Maruta M, Utsumi T, et al. Bladder and male sexual functions after autonomic nerve-sparing TME with or without lateral node dissection for rectal cancer. Tech Coloproctol 2003; 7:29-33.

5. Matsumoto T, Ohue M, Sekimoto M, et al. Feasibility of autonomic nerve-preserving surgery for advanced rectal cancer based on analysis of micrometastases. Br J Surg 2005; 92:1444 8.

6. Moriya Y, Sugihara K, Akasu T, et al. Patterns of recurrence after nerve-sparing surgery for rectal adenocarcinoma with special reference to loco-regional recurrence. Dis Colon Rectum $1995 ; 38: 1162-8$.

7. Shirouzu K, Ogata Y, Araki Y. Oncologic and functional results of total mesorectal excision and autonomic nerve-preserving operation for advanced lower rectal cancer. Dis Colon Rectum 2004; 47:1442-7.

8. Sugihara K, Moriya Y, Akasu T, et al. Pelvic autonomic nerve preservation for patients with rectal carcinoma. Oncologic and functional outcome. Cancer 1996; 78:1871-80.

9. Tsunoda A, Shibusawa M, Tanizaki H, et al. Hypogastric nerve preservation does not increase local recurrence after surgical treatment of rectal carcinoma. Hepatogastroenterology 2004; 51:1354-7.

10. Kim NK, Aahn TW, Park JK, et al. Assessment of sexual and voiding function after total mesorectal excision with pelvic autonomic nerve preservation in males with rectal cancer. Dis Colon Rectum 2002; 45:1178-85.
11. Moriya Y. Function preservation in rectal cancer surgery. Int $J$ Clin Oncol 2006; 11:339-43.

12. Platell CF, Thompson PJ, Makin GB. Sexual health in women following pelvic surgery for rectal cancer. Br J Surg 2004; 91:465-8.

13. Liang JT, Lai HS, Lee PH. Laparoscopic pelvic autonomic nerve-preserving surgery for patients with lower rectal cancer after chemoradiation therapy. Ann Surg Oncol 2007; 14:12857.

14. Liang JT, Chang KJ, Wang SM. Anatomical basis of autonomic nerve-preserving total mesorectal excision for rectal cancer. Br J Surg 1997; 84:586-7.

15. Liang JT, Chien CT, Chang KJ, et al. Neurophysiological basis of sympathetic nerve-preserving surgery for lower rectal cancer-a canine model. Hepatogastroenterology 1998; 45:220614.

16. Liang JT, Chang KJ, Wang SM. Lateral rectal ligaments contain important nerves. Br J Surg 1998; 85:1162.

17. Walsh PC, Schlegel PN. Radical pelvic surgery with preservation of sexual function. Ann Surg 1988; 208:391-400.

18. Leroy J, Henri M, Rubino F, et al. Sigmoidectomy. In: Milsom J, Böhm B, Nakajima K, editors. Laparoscopic Colorectal Surgery. New York: Springer-Verlag 2006; 145-69.

19. Liang JT, Lai HS, Lee PH. Oncologic results of laparoscopic versus conventional open surgery for stage II or III left-sided colon cancers: a randomized controlled trial. Ann Surg Oncol 2007; 14:109-17.

20. Jayne DG, Brown JM, Thorpe H, et al. Bladder and sexual function following resection for rectal cancer in a randomized clinical trial of laparoscopic versus open technique. Br J Surg 2005; 92:1124-32.

21. Rosen RC, Riley A, Wagner G, et al. The International Index of Erectile Function (IIEF): a multidimensional scale for assessment of erectile dysfunction. Urology 1997; 49:822-30.

22. Rosen R, Brown C, Heiman J, et al. The Female Sexual Function Index (FSFI): a multidimensional self-report instrument for the assessment of female sexual function. $J$ Sex Marital Ther 2000; 26:191-208.

23. Liang JT, Lai HS, Huang KC, et al. Comparison of medial-tolateral versus traditional lateral-to-medial laparoscopic dissection sequences for resection of rectosigmoid cancers: randomized controlled clinical trial. World J Surg 2003; 27:190-6.

24. da Silva GM, Zmora O, Börjesson L, et al. The efficacy of a nerve stimulator (CaverMap) to enhance autonomic nerve identification and confirm nerve preservation during total mesorectal excision. Dis Colon Rectum 2005; 48:2354-61. 